\title{
QIS FOR APPLIED QUANTUM FIELD THEORY
}

Fermilab: Marcela Carena (PI), Joshua Isaacson, Roni Harnik, Ciaran Hughes, Andreas Kronfeld, Henry Lamm, Alexander Macridin, Panagiotis Spentzouris, James Simone, Michael Wagman Caltech: John Preskill, Alex Buser, Junyu Liu, Ashley Milsted

Institute For Nuclear Theory: David B. Kaplan, Martin J. Savage, Silas R. Beane, Stephan Casper Anthony Ciavarella, Natalie Klco, Jesse Stryker

Precision predictions in HEP and non-equilibrium dynamics in the early universe require quantum computational technologies. Future quantum devices offer the possibility of performing these, complementing lattice quantum chromodynamics (QCD) studies $\begin{aligned} & \text { Figure 1: Event in the CMS Experiment detector from } 7 \\ & \text { TeV collisions. (Image credit: CMS) }\end{aligned}$ on classical computers.

We are investigating many aspects of the quantum simulation of quantum field theories. Specific topics include simulation-suitable formulations of gauge theories, state preparation, time evolution, extracting HEP-related observables from simulations, quantum error correction (QEC), and entanglement. We are exploring strategies for simulation of scattering in QFTs, and dynamics of the early Universe.

\section{Formulations of Quantum Field Theories}

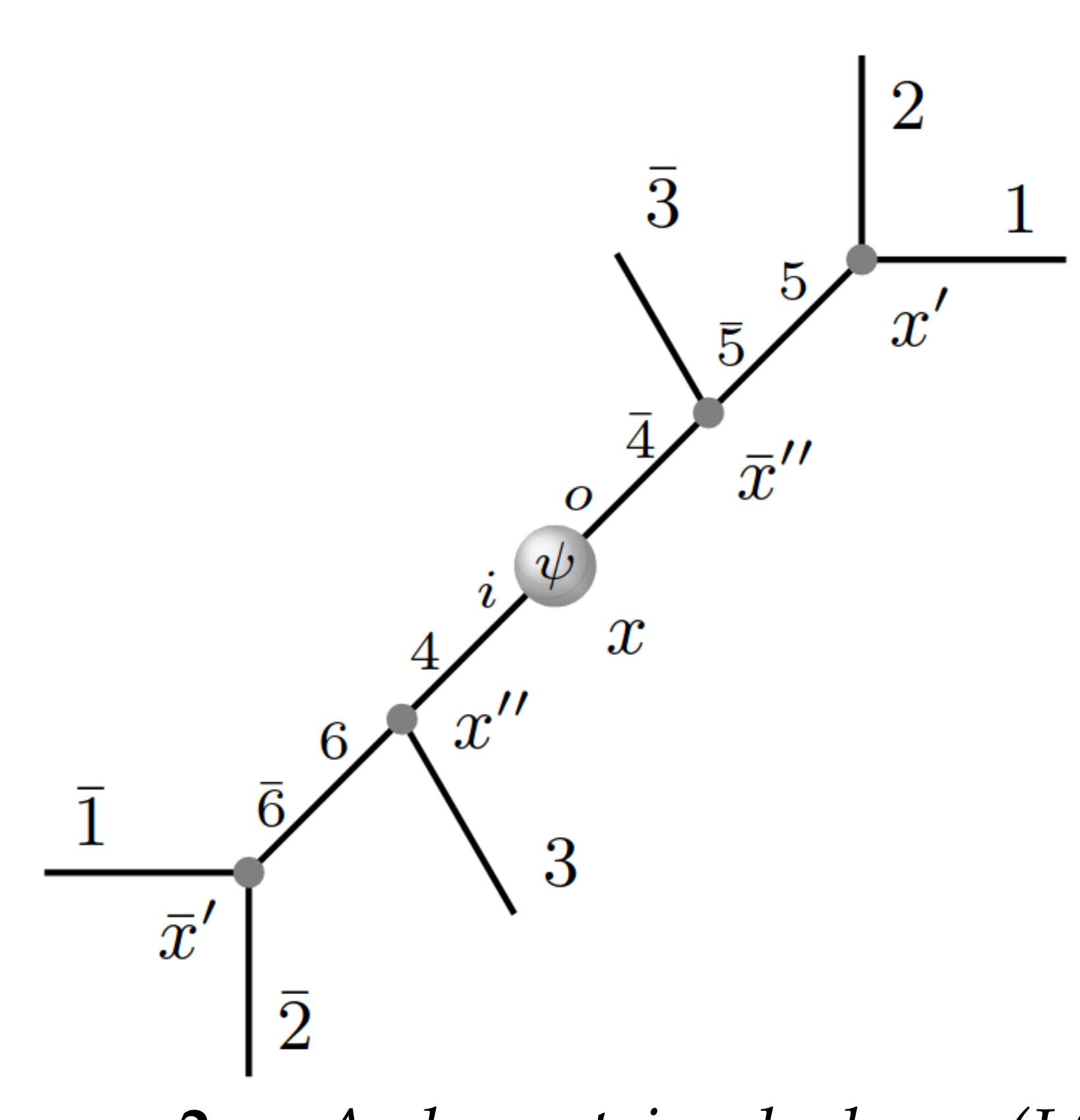

Figure 2: A loop-string-hadron ( $\mathrm{LSH}$ ) formulation for SU(2) Hamiltonian lattice gauge theory (1)

Efficient choices of basis, truncation, and quantum gate implementation are critical, particularly in the NISQ era. Our team has developed several promising options for simulating both gauge (1-3) and scalar theories $(4,5)$. As these options mature, resource cost estimates and comparisons can be made.

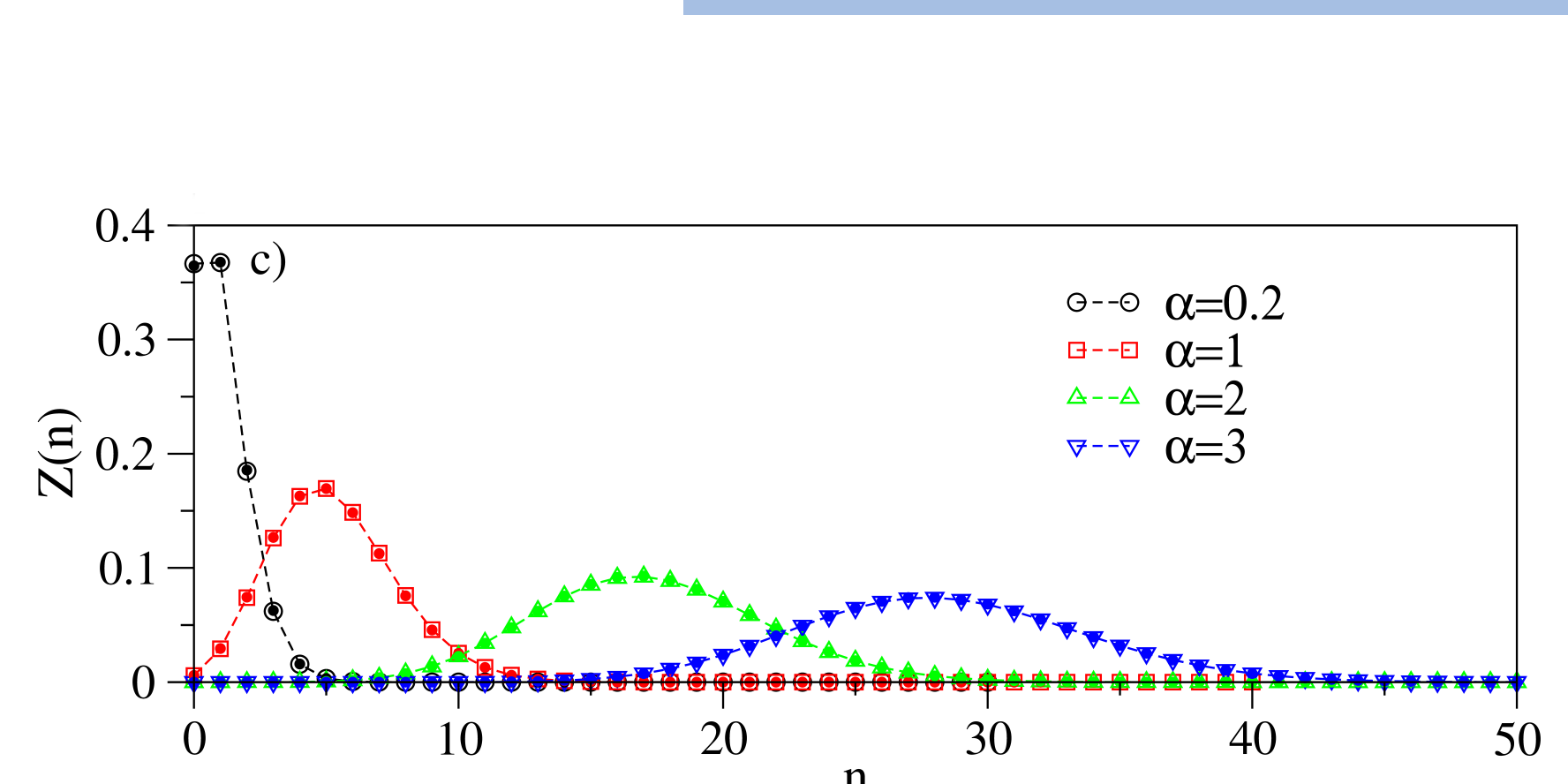

Figure 4: The phonon number distribution in the polaron state for different values of the coupling strength (4).
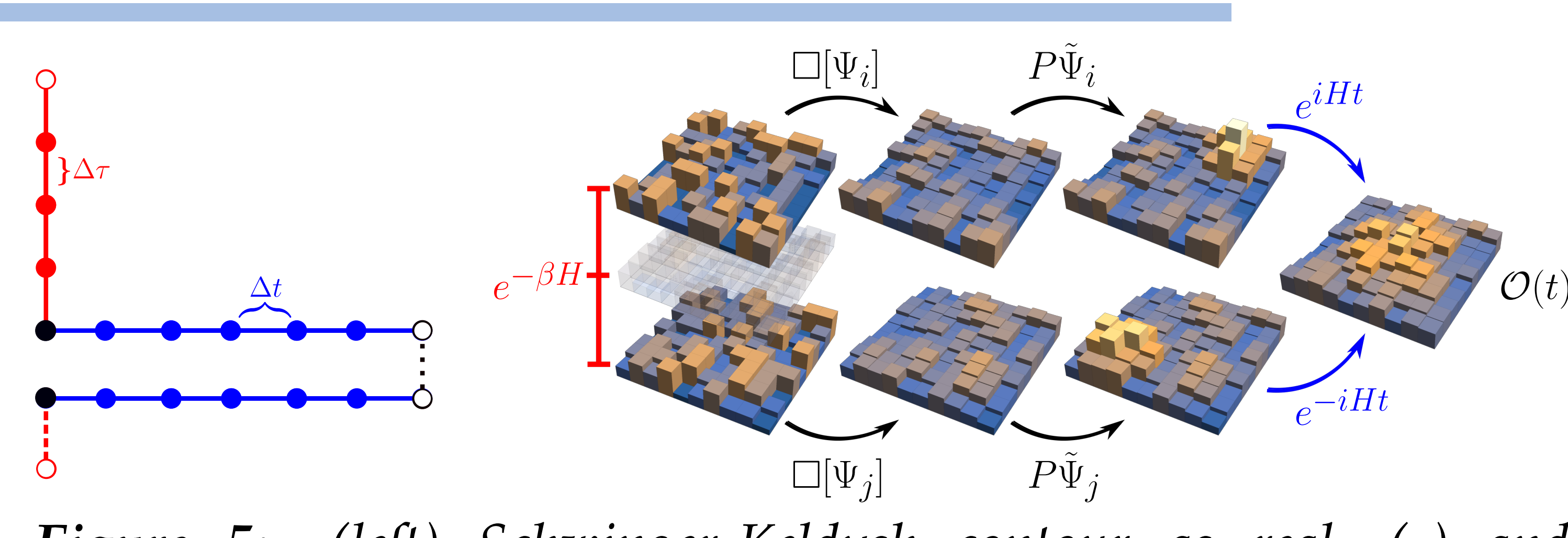
matched, at (०) one inserts $\mathcal{O}$ (right) how smearing, $\square[\Psi]=\tilde{\Psi}$, and
sources $P$ allow general state preparation (6)
Figure 5: (left) Schwinger-Keldysh contour as real- (๑) and imaginary-time (•) path integrals. At (•) two path integrals are

\section{State Preparation}

Leveraging renormalization group and lattice QCD ideas, new state preparation

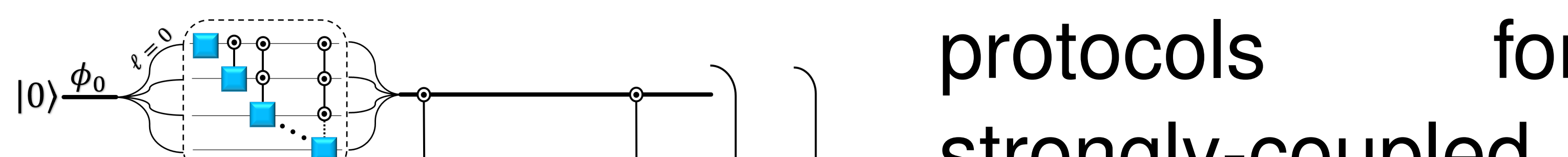
${ }_{100} \phi_{1}$

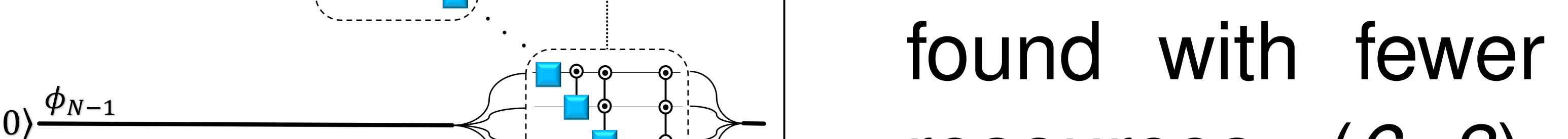
(0) $\frac{\phi_{N-1}}{3}$ ? resources (6-8). Figure 6: Quantum circuit for prepar- Work is ongoing ing an arbitrary real wavefunction for a with
scala fietd initialize a scalar theory ground state.

\section{Simulating Time Evolution of QFTs}

We are continuing to develop algorithms for time evolution of QFTs. Simulations of a truncated, low-dimensional non-Abelian gauge theory were performed with IBM (3).

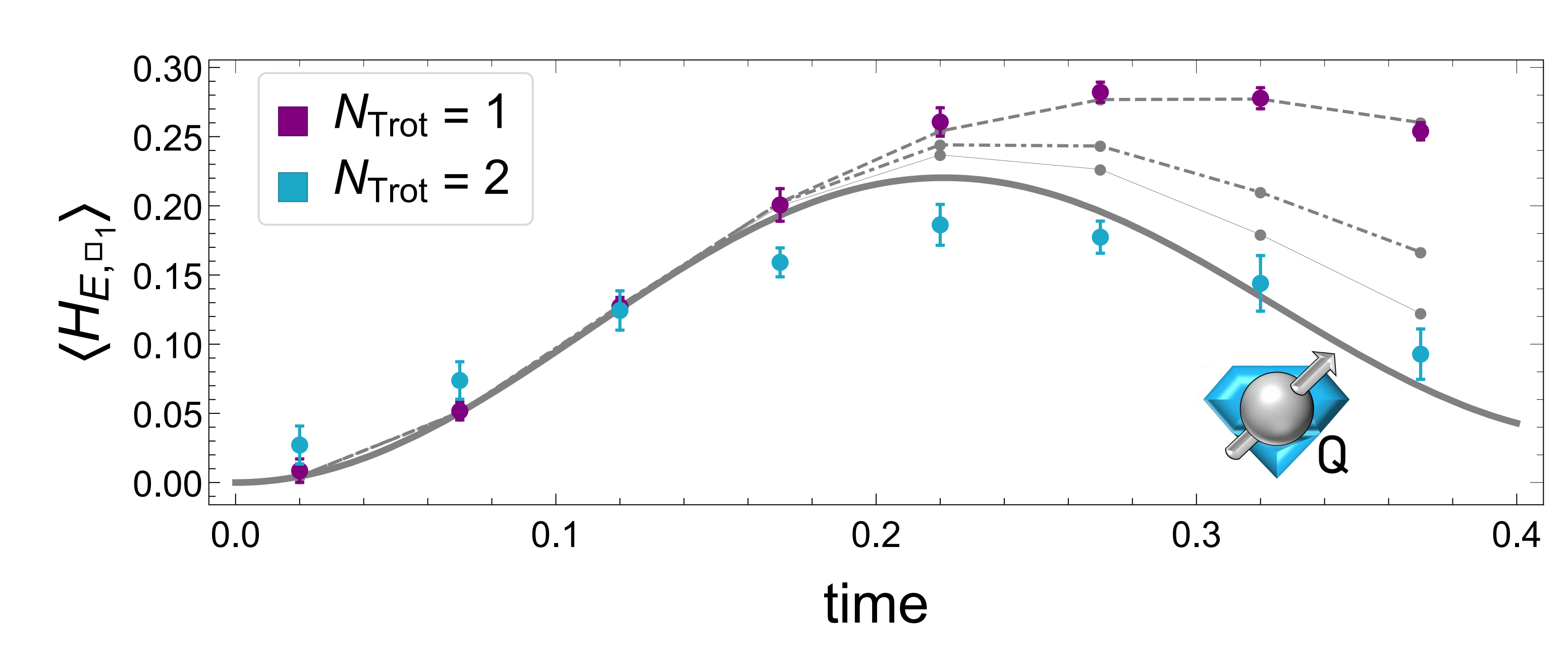

Figure 7: Expectation value of the electric energy contribution of the first plaquette in a two-plaquette non-Abelian lattice field theory using

\section{Quantum Error Correction}

Error correction is required for precision predictions from QFT. Studies have started of the interplay of QEC and continuous symmetries that arise in QFT (9).

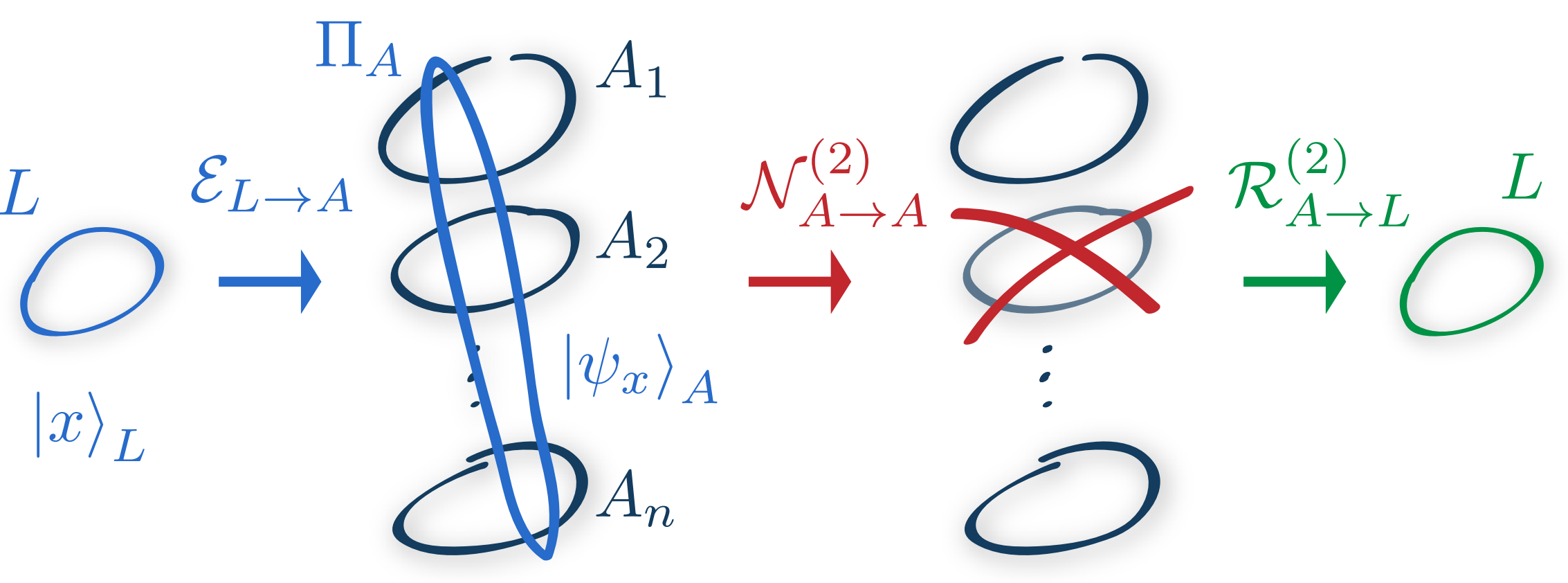

Figure 8: A code $\mathcal{E}_{L \rightarrow A}$ maps a logical state $|x\rangle$ to a physical state $\left|\psi_{x}\right\rangle_{A}$
composed of several subsystems $A=A_{1} \otimes \cdots A_{n}$. While a subsystem can be erased, $\mathcal{N}_{A \rightarrow A}^{i}$, a good error-correcting code recovers the original logical state $|x\rangle$ by applying $\mathcal{R}_{A \rightarrow L}^{i}(9)$

\section{Entanglement and QCD}

QIS-inspired insight has lead to the discovery of new properties related to entanglement in nonperturbative QCD $(10,11)$

\section{Observables for Colliders}

$0.2::_{m=1.5, g=0.0}^{m=1.4, g=0.4}$

We have shown how \& 0.1 to compute parton distribution functions (12) and how many prop-

0.05 $\begin{array}{lllllll}0 & 0.2 & 0.4 & 0.6 & 0.8 & 1\end{array}$ erties can be obtained with few measureFigure 9: PDF of a 1+1d ments (13). These fermionic theory obtained from a will be extended to
quantum simulator (12) fragmentation functions and viscosity.

\section{Outlook: Simulating Cosmology}

Our methods can be extended to early universe processes like: QFTs in curved spacetimes, bubble collisions, and CP-violating transmissions of fermions off bubbles.

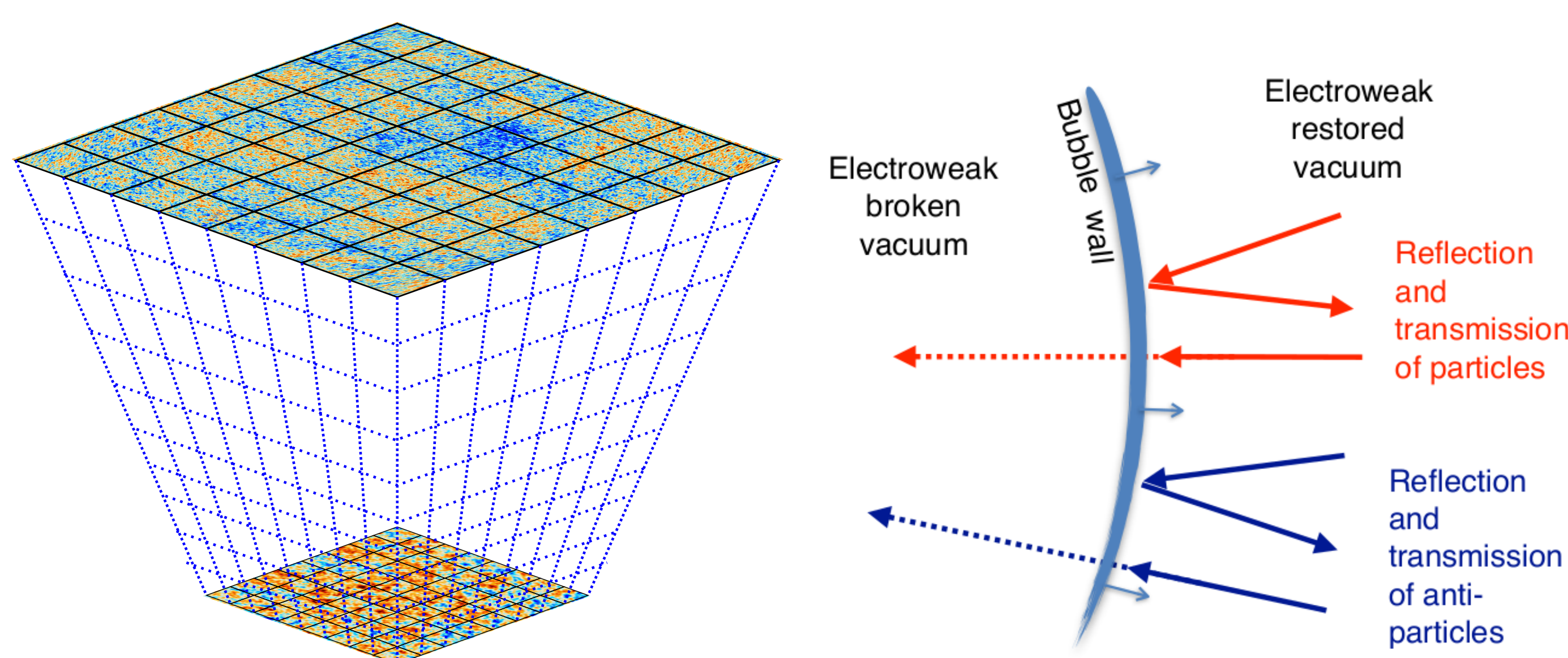

Figure 10: We are developing formalism for (left) QFTs in expanding backgrounds and (right) baryogenesis in the expanding universe.

pt has been authored by Fermi Research Alliance, LLC under Contract No. DEAC02-07CH1 1359 with the U.S. Department of Energy, Office of Science, Office of High Energy Physics.

1. I. Raychowchury, J. R. Stryker, arxiv: 1912.06133 (hep-lat) D. . . Hackett et al, Phys. Rev.A 99, 062341 (2019).

4. A. Macridin, P. Spentzouris, J. Amundson, R. Harnik, Phys. Rev. Lett. 121, 110504 (2018). 5. N. Klco, M. J. Savage, Phys.Rev.A 99, 052335 (2019).

6. S. Harmalkar, H. Lamm, S. Lawrence, arXiv: 200

7. N. Klco, M. J. Savage, arXiv: 2002.02018 (quant-ph)

8. N. Klco, M. J. Savage, arXiv: 19

S. R. Beane, D. B. Kaplan, N. Klco, M. J. Savage, Phys.Rev.Lett. 122, 102001 (2019).

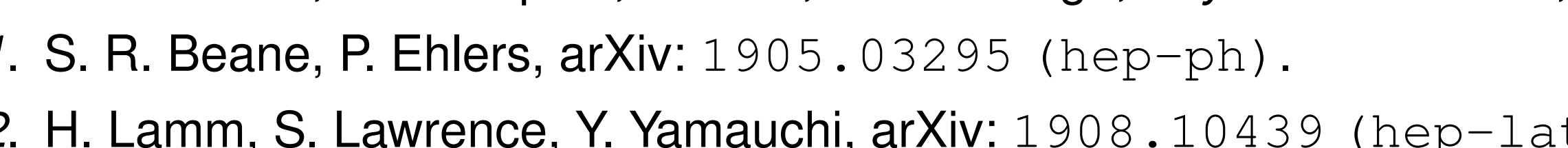

. H-Y.Huang R. Kueng, J. Proskill, 2020, arXiv: 2002.08953 (quant-ph). 